\title{
Dalla medicina reattiva alla medicina di precisione
}

\author{
Sandra La Rosa ${ }^{1}$, Chiara Guglielmo ${ }^{1}$, Alessandra Ocello ${ }^{1}$, Concetto Sessa ${ }^{2}$, Giuseppe Seminara ${ }^{3}$, Antonio Granata ${ }^{3}$ \\ ${ }^{1}$ U.O. Nefrologia e Dialisi, P.O. "Giovanni Paolo II", Sciacca (AG) - Italy \\ ${ }^{2}$ U.O.C Nefrologia e Dialisi, P.O. "Maggiore", Modica (RG) - Italy \\ ${ }^{3}$ U.O.C. Nefrologia e Dialisi Azienda Ospedaliera Cannizzaro, Catania (CT) - Italy
}

\begin{abstract}
From reactive medicine to precision medicine
In recent years, there has been increased awareness of a concept of medicine based on individual differences taking into consideration genetic variability, environment, characteristics of the microbiome and individual lifestyles. It makes use of genomics, transcriptomics, proteomics and metabolomics techniques, obtaining a large amount of information which enables a more precise characterization of the patient. This model expands to the principles of prediction, prevention, personalization and participation, including all medical specialties. In nephrology, the application of precision medicine could play a central role, thanks to the information available today in multiple fields: for example the impact of alterations in the intestinal microbiota on the progression of chronic renal failure, in polycystic disease, in diabetic nephropathy and in the personalized approach to the transition period before the beginning of hemodialysis therapy.
\end{abstract}

Keywords: Genomics, Patient characterization, Personalized medicine, Precision medicine, Transcriptomics

\section{Introduzione}

"Tutto comincia con l'individuo", affermava Carl Gustav Jung, e in una società che è sempre più concentrata sui bisogni individuali e che punta alla personalizzazione dei servizi anche la medicina sembra assumere un'inclinazione soggettiva. Negli ultimi anni, si sta diffondendo sempre di più un concetto di medicina "cucito", "tailleurizzato" sulle differenze individuali, che tiene conto della variabilità genetica, dell'ambiente, delle caratteristiche del microbioma e dello stile di vita delle singole persone. II sequenziamento del genoma umano ha avviato un nuovo modo di indagare i meccanismi cellulari. La genomica, la transcrittomica, la proteomica e la metabolomica hanno prodotto una grande quantità di informazioni, che permettono una sempre più precisa caratterizzazione del paziente (1).

In passato, la medicina era praticata avendo come unici riferimenti i segni e i sintomi del paziente, basandosi esclusivamente sulle conoscenze individuali del medico, e, per questo, veniva chiamata medicina dell'intuizione o reattiva. Attualmente, la medicina è basata sui dati prodotti dalla

Received: July 29, 2021

Accepted: August 3, 2021

Published online: September 18, 2021

Indirizzo per la corrispondenza:

Sandra La Rosa

Unità Operativa di Nefrologia e Dialisi

Ospedale "Giovanni Paolo II"

Via Pompei

92019 Sciacca (AG) - Italy

slarosa76@yahoo.it ricerca scientifica, raccolti con studi randomizzati e controllati, ed è inquadrata come medicina basata sull'evidenza (2). In futuro, la medicina potrebbe seguire degli algoritmi che prendano in considerazione selettivamente le caratteristiche genetiche e fenotipiche dei singoli pazienti, costituendo, così, la medicina di precisione (3) (Fig. 1).

Questo potrebbe essere un nuovo modo di pensare la medicina, un modo che prevede l'utilizzo di tecnologie di profiling molecolare per migliorare l'accuratezza diagnostica, la stima della prognosi e la scelta della giusta strategia terapeutica per il paziente giusto al momento giusto (4). II concetto della "medicina personalizzata" era stato anticipato nel tardo 1800 dal medico Canadese William Osler, che aveva notato la grande variabilità esistente tra individui diversi (5). In seguito, la farmacogenetica è divenuta la principale area di applicazione della medicina personalizzata e, sebbene le prime osservazioni di reazioni inusuali ai farmaci basate sull'individualità biochimica fossero state notate nel 1930, la prima pubblicazione riguardante la farmacogenetica risale al 1959 ad opera del medico tedesco Friedrich Vogel (6). Negli anni, vi è stato un progressivo incremento delle pubblicazioni riguardanti tale argomento, ma l'interesse intorno al tema della medicina di precisione è esploso solo recentemente.

Prendendo spunto dal passato, la medicina nel ventunesimo secolo dovrebbe concentrarsi sul raggiungimento delle " $4 P$ " teorizzate da Pulciani et al (7), predizione, prevenzione, personalizzazione e partecipazione, con l'obiettivo di istituire il percorso di salute per ogni singolo individuo utilizzando il suo profilo molecolare e cercando di determinare il miglior intervento medico per mantenere o ripristinare lo stato di benessere e salute. 


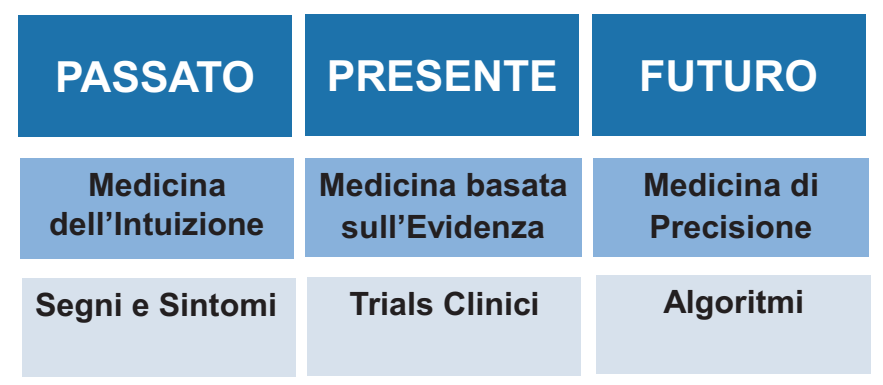

Fig. 1 - L'evoluzione della Medicina.

Queste metodologie di studio sono state applicate in campo clinico con tre finalità: 1) la caratterizzazione dei fenotipi patologici, attraverso l'identificazione di pattern di espressione che discriminano il malato dal sano; 2 ) la determinazione dei profili predittivi di patologia; 3) l'identificazione delle caratteristiche metaboliche individuali che possano indirizzare il clinico a prevedere l'efficacia o la tossicità di un trattamento farmacologico.

Mentre la medicina reattiva prevede di iniziare a trattare le malattie dopo la loro manifestazione clinica, agire prima della comparsa dei sintomi è il modus operandi della medicina di precisione (8). Gli interventi preventivi si focalizzano sui fattori di rischio associati alle patologie (come, per esempio, tabagismo e tumore polmonare) e generano terapie necessarie a prevenire l'evento malattia (9). II termine "precisione" viene utilizzato perché si vuole riuscire a classificare le persone in sottopopolazioni usando il loro corredo genetico, gli stili di vita, le risposte terapeutiche e i fattori culturali e ambientali che li accomunano (10).

Nel 1990, è stato creato il Progetto del genoma umano, un progetto internazionale, mirato a caratterizzare il completo set di informazioni genetiche di ogni singolo essere umano, che ha permesso, nel 2003, la completa mappatura dei geni del genoma umano anche grazie alla tecnica del positional cloning (11). Tutto ciò oggi permette che la ricerca parta dall'osservazione del paziente e dalla descrizione della malattia, per proseguire in un laboratorio di ricerca, in cui la tecnologia del DNA ricombinante e quella dell'ingegneria genetica vengono estensivamente impiegate per individuare il gene mutato (Fig. 2).

Tenendo in considerazione la necessità di riorganizzare le patologie in relazione al loro aspetto biomolecolare, il Consiglio Nazionale per la Ricerca ha suggerito la creazione di un nuovo sistema di tassonomia (12) che integri informazioni cliniche e dati di ricerca riguardanti il genoma, il microbioma, l'epigenoma, l'esposoma e segni e sintomi di specifiche popolazioni.

\section{Applicazioni della medicina di precisione partendo dall'oncologia}

Sebbene intervengano numerosi fattori nell'eziologia del cancro, il profondo legame tra genoma e neoplasie (13) ha dato un input importante per lo sviluppo della medicina di

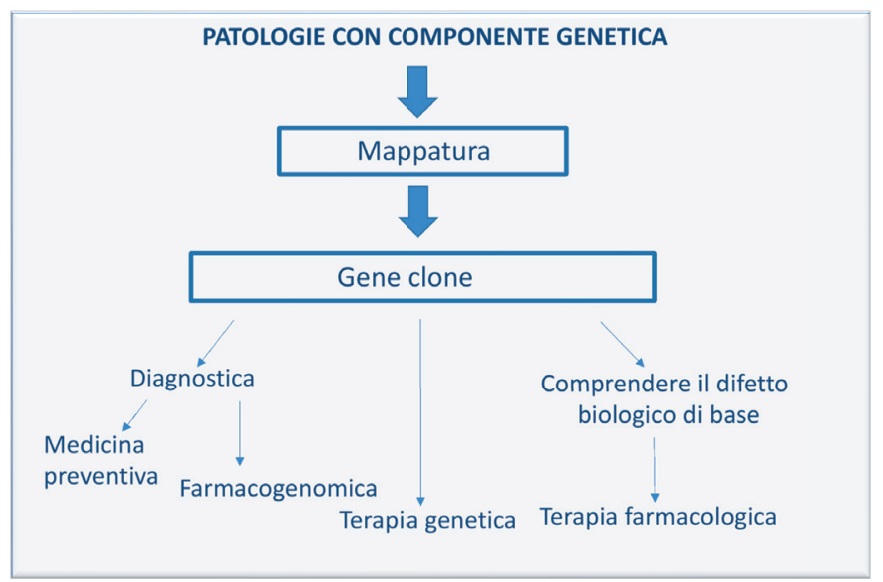

Fig. 2 - Mappatura genetica e terapia.

precisione. Farmaci con azione mirata verso kinasi oncogeniche, che includono i recettori tirosin-protein kinasi erB-2 (HER2), BRAF o EGFR (epidermal growth factor receptor), hanno dimostrato una risposta clinica in specifici gruppi di pazienti. L'espressione incontrollata di HER2 è associata con una forma aggressiva di tumore alla mammella con prognosi sfavorevole. II trattamento con trastuzumab, un anticorpo monoclonale diretto verso HER2, ha mostrato benefici in termini di sopravvivenza, con una riduzione del $20 \%$ del rischio di morte a 30 mesi (14). L'attivazione di mutazioni in BRAF, presenti nella metà dei casi di melanoma, rappresenta il target di uno specifico inibitore quale il vemurafenib (15). Anticorpi monoclonali anti-EGFR sono diventati farmaci di prima linea per il tumore polmonare non a piccole cellule e riescono a garantire una migliore qualità di vita e una sopravvivenza più lunga libera da progressione di malattia (16). In aggiunta, la medicina di precisione prevede il trattamento con poli ADPribosio polimerasi (PARP) per i tumori contenenti mutazioni di BRCA1 o BRCA2 come il tumore ovarico (17).

Partendo dall'oncologia, i progressi riguardanti la medicina di precisione sembrano destinati a estendersi anche alla nefrologia che, per quanto inizialmente non fosse considerata la disciplina attorno a cui si era sviluppata la medicina di precisione, potrebbe avere un ruolo centrale grazie ai dati disponibili sull'impatto delle alterazioni del microbiota intestinale sulla progressione dell'insufficienza renale cronica, sulla nefropatia diabetica, sui reni policistici, sulla nefropatia diabetica e sull'approccio personalizzato nel periodo di transizione che porta all'inizio della terapia emodialitica.

L'uso di tecnologie di sequenziamento di nuova generazione (NGS) nella pratica clinica fornisce una visione più approfondita dei meccanismi patologici delle varie malattie renali (Tab. I). II NGS ha contribuito alla scoperta di nuove cause genetiche per una varietà di fenotipi clinici, come le ciliopatie renali, le anomalie congenite del rene e del tratto urinario (CAKUT), la glomerulosclerosi segmentale focale, la sindrome nefrosica e la sindrome emolitico-uremica. 
TABELLA I - Principali alterazioni genetiche di alcune patologie renali

\begin{tabular}{ll}
\hline $\begin{array}{l}\text { Analisi molecolare mediante NGS } \\
\left({ }^{*}\right) \text { di alcune patologie renali }\end{array}$ & $\begin{array}{l}\text { Principali alterazioni genet- } \\
\text { iche }\end{array}$ \\
\hline $\begin{array}{l}\text { Rene Policistico Autosomico } \\
\text { Dominante }\end{array}$ & PKD1, PDK2 \\
Rene Policistico Recessivo & PKHD1 \\
Nefronoftisi & NPHP1, NPHP2, NPHP3, \\
Sindrome di Alport & COL4A3, COL4A4, COL4A5 \\
Sclerosi Tuberosa & TSC1, TSC2 \\
Glomerulosclerosi Segmentale & ACTN4, PAX2, CD2AP, TRPC6 \\
Focale & \\
Malattia di Fabry & GLA \\
Sindrome Nefrosica & NPHS1, NPHS2, WT1 \\
Cakut & DSTYK \\
Nefropatia Iperuricemica & UMOD \\
Acidosi Tubulare & AT6VOA4, ATP6V1B1, SLC4A1 \\
Sindrome Emolitico-uremica & DGKE \\
Sindrome di Liddle & SCNN1B, SCNN1G \\
\hline
\end{tabular}

\section{Attuali sviluppi della medicina di precisione}

\section{- Impatto delle alterazioni del microbiota intestinale sulla progressione della malattia renale cronica}

L'insufficienza renale cronica associata all'uremia è aggravata dalla disbiosi. L'intestino umano ospita più di 100 miliardi di batteri, costituendo un serbatoio di oltre 1 grammo di endotossine. In condizioni di normalità, il microbiota intestinale può generare tossine uremiche che sono generalmente riassorbite ed eliminate dai reni. Nella malattia renale cronica, l'afflusso di urea, acido urico e ossalati dal tratto intestinale aumenta con il peggioramento della funzione renale, che può alterare il bilancio tra commensali e patogeni e che porta alla disbiosi intestinale (18). Nei pazienti con malattia renale cronica, sono state evidenziate, infatti, alterazioni della composizione del microbiota rispetto ai soggetti sani, con incremento di Proteobacteria, Enterobacteriacee e Clostridium, a discapito di Lactobacilli e Bifidobacteria. Alla disbiosi consegue un incremento del metabolismo proteolitico e della produzione di tossine uremiche, che aumentano anche per riduzione dell'escrezione renale (19). Sono stati identificati specifici batteri intestinali, come Oscillibacter, Lacnospira e Veillonella, la cui concentrazione in maniera abbondante è riscontrata nei pazienti affetti da malattia renale cronica rispetto ai soggetti controllo. L'Oscillibacter, in particolar modo, sembra creare un ambiente favorevole per la produzione di vari metaboliti uremici (20).

L'indossil solfato e il p-cresolo solfato sono generati dalla fermentazione batterica di una dieta proteica, così come anche la trimetilammina, trasformata, a livello epatico, in trimetilammina- $\mathrm{N}$-ossido (TMAO) (21). II p-cresolo e l'indossil solfato sono stati correlati a un'aumentata mortalità nei pazienti con malattia renale cronica e TMAO, noto fattore aterogeno, è stato associato a un aumento di eventi cardiovascolari. Su queste tossine, sensibilmente aumentate nei pazienti con malattia renale cronica, agiscono inibitori della biosintesi batterica del colon, sostanze che ne inibiscono l'assorbimento e che aumentano la loro clearance, tra cui probiotici e prebiotici. I probiotici sono composti da organismi viventi ingeriti attraverso alimenti o integratori, mentre i prebiotici sono carboidrati non digeribili che stimolano l'attività dei batteri intestinali. I probiotici, come bifidobacterium species, lattobacilli e streptococchi, possono influenzare la risposta infiammatoria. Il trattamento con lattobacilli può ristabilire la funzione della barriera intestinale. I pazienti in emodialisi trattati con dosi orali di Lattobacillo Acidofilo hanno un decremento nel siero di dimetilammina, una potenziale tossina uremica. La Figura 3 riassume le recenti applicazioni dei prebiotici e dei probiotici per attenuare i disturbi del microbioma intestinale nelle patologie renali.

\section{- La medicina di precisione e la malattia policistica renale}

La malattia policistica renale autosomica dominante (ADPKD) è la patologia ereditaria più frequentemente causa di malattia renale cronica. La mutazione in almeno due geni, PKD1 e PKD2, è causa di una ridotta funzione delle loro rispettive proteine, policistina 1 e policistina 2 (22), e ciò causa, nelle cellule tubulari renali, una riduzione delle concentrazioni di calcio intracellulare e un aumento dei livelli di adenosina monofosfato ciclico (23) (Fig. 4). I farmaci per la cura di questa patologia attualmente in commercio, Tolvaptan, analogo della somatostatina e inibitori di mTOR, sono esempi di medicina di precisione.

II Tolvaptan è un antagonista selettivo per il recettore V2 dell'ormone vasopressina presente nel tubulo renale distale e nel dotto collettore; in questa sede, Tolvaptan induce una riduzione dei livelli di adenosina monofosfato ciclico (cAMP) (elevati livelli di cAMP causano proliferazione cellulare e passaggio dello ione sodio e di acqua nello spazio extracellulare) (24). Il Tolvaptan rallenta la proliferazione cellulare delle cisti, la secrezione di fluido all'interno delle cisti e il declino della funzione renale nei pazienti con ADPKD con malattia renale cronica agli stadi da 1 a 3, con evidenza di malattia in rapida progressione. La riduzione dell'incremento volumetrico dei reni è particolarmente spiccata nel primo anno di trattamento e persiste nel follow-up (25).

Per quanto riguarda la somatostatina, è un peptide endogeno secreto dalle isole di Langerhans (cellule delta), dal tratto gastro-intestinale, dall'ipotalamo e dalla tiroide (26); interagisce con recettori accoppiati a proteine G (SSTRs 1-5) e determina un'inibizione dell'attività dell'enzima adenilato ciclasi, della proliferazione cellulare, della secrezione 


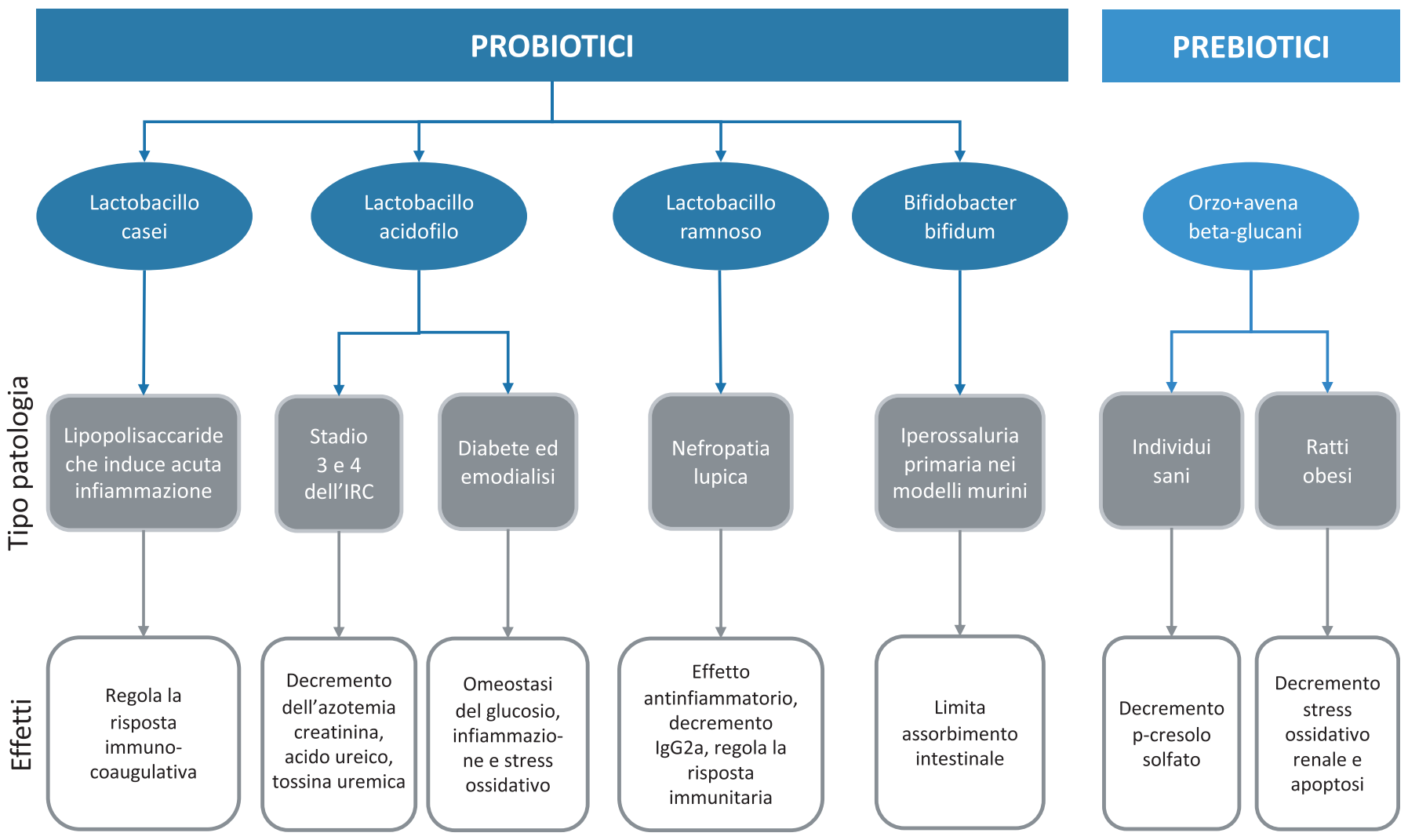

Fig. 3 - Applicazione dei prebiotici e dei probiotici nelle patologie renali.

di numerosi ormoni (ormone della crescita, insulina, glucagone, gastrina) e di fattori di crescita (fattore di crescita similinsulinico 1 e fattore di crescita dell'endotelio vascolare) (27).
Diversamente da Tolvaptan, la cui efficacia è limitata alle cellule del tubulo distale e del dotto collettore, gli analoghi della somatostatina, tra cui anche l'Octreotide (28), legandosi ai

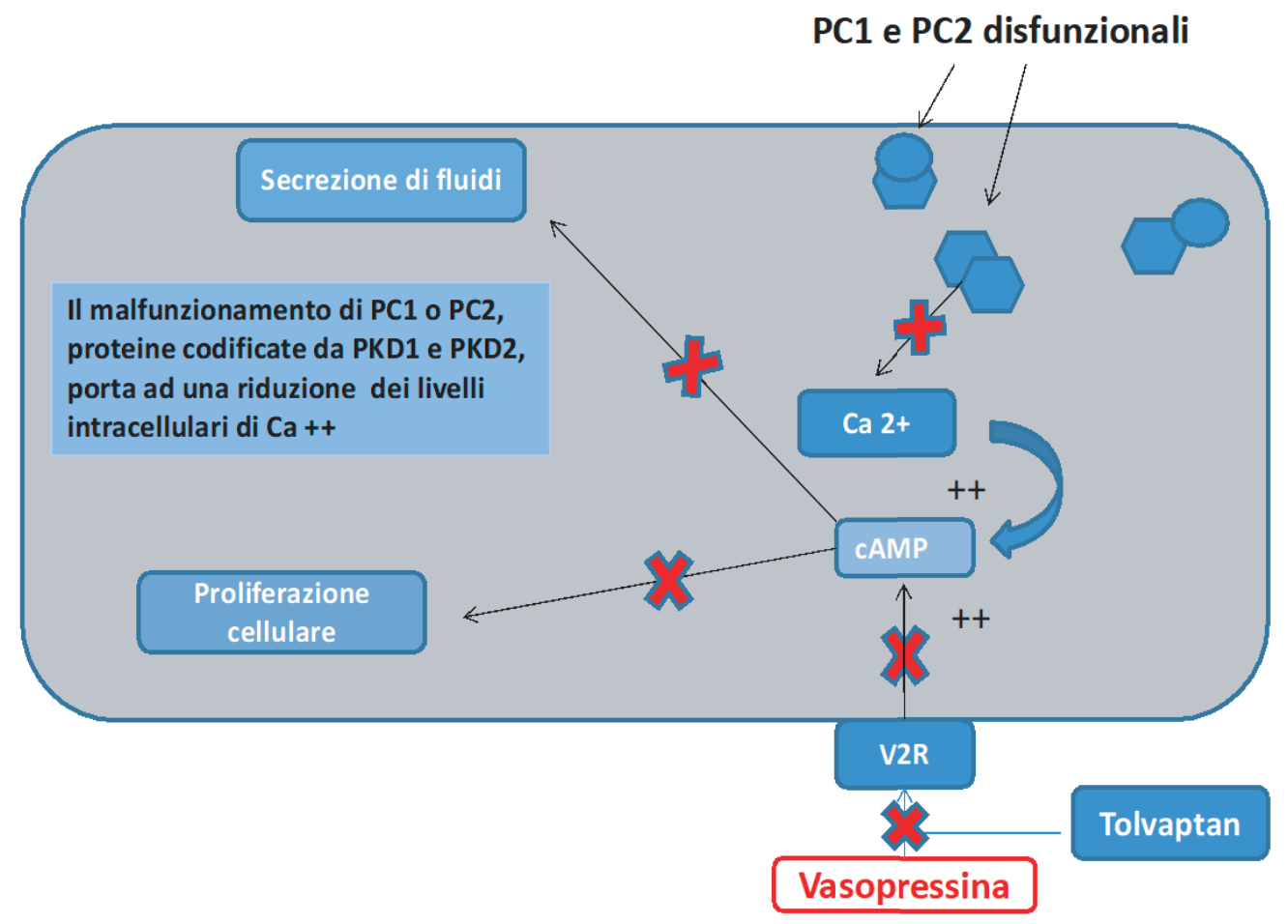

Fig. 4 - Applicazione del Tolvaptan. 
recettori presenti anche sul tubulo prossimale, agiscono su un numero maggiore di cisti (29). Recentemente, l'European Medicines Agency ha stabilito che l'analogo della somatostatina, Lanreotide, debba essere considerato farmaco "orfano" per il trattamento dell'ADPKD.

La serina/treonina chinasi Mammalian Target of Rapamycin ( $m$ TOR) è un enzima che svolge un ruolo importante nell'ambito dei meccanismi di regolazione della proliferazione cellulare (30). La policistina 1, formando un complesso con la tuberina, agisce come inibitore endogeno di mTOR. Il deficit genetico o di funzione di policistina 1, tipico dell'ADPKD, comporta un'attivazione aberrante di mTOR (31). In base a questi dati è stato suggerito un possibile ruolo terapeutico degli inibitori di mTOR nell'ADPKD per i potenti effetti antiproliferativi e antifibrotici (32). Gli inibitori di mTOR risultano sicuri e riducono l'incremento del volume renale, ma, recentemente, è stato dimostrato che non hanno alcun effetto sul rallentamento del declino della velocità di filtrazione glomerulare stimata (eGFR) e si associano a un aumento della proteinuria. Per tale motivo, non risulta ancora possibile proporre questo trattamento nella pratica clinica (33).

\section{- La medicina di precisione e la nefropatia diabetica}

Il diabete mellito tipo 2 è la causa principale della malattia renale cronica. Nel 2013, quasi 3,4 milioni di italiani hanno avuto una diagnosi di diabete, più del doppio rispetto al 1980. La prevalenza negli uomini è passata dal 3,3\% del 1980 al $7,1 \%$ del 2013 e, nelle donne, dal 4,7\% al 6,8\% (34).

Secondo le più recenti stime epidemiologiche, nel 2017 in tutto il mondo circa 425 milioni di adulti di età compresa tra i 20 e i 79 anni erano affetti da diabete mellito (35). Tale incremento è dovuto sia all'invecchiamento della popolazione sia ad altri fattori, tra cui la diagnosi precoce e l'aumento della sopravvivenza dei malati di diabete grazie al miglioramento delle terapie.

La medicina di precisione prevede l'utilizzo delle glifozine, inibitori del co-trasportatore sodio-glucosio (SGLT), che, oltre a ridurre in maniera significativa la progressione della nefropatia diabetica verso gli stadi più avanzati dell'insufficienza renale, riducono anche il rischio di morte cardiovascolare e di ospedalizzazione per scompenso cardiaco (36).

Vi sono due tipologie di co-trasportatori sodio-glucosio: SGLT2, localizzato nel tubulo contorto prossimale, e SGLT1, situato più a valle nella parte terminale del tubulo contorto prossimale e anche nell'intestino tenue. II co-trasportatore SGLT2 è responsabile del riassorbimento del $90 \%$ del glucosio e SGLT1 del restante $10 \%$.

Dapaglifozin ed Empaglifozin, i primi farmaci inibitori del co-trasportatore sodio-glucosio a essere stati immessi in commercio in Italia, inibiscono selettivamente SGLT1 e 2, riducendo la soglia plasmatica renale per il glucosio e inducendo glicosuria. Altri effetti di questi farmaci sono la riduzione del peso corporeo e della pressione arteriosa, il calo degli episodi di ipoglicemia e l'aumento del rischio di infezioni genitali e urinarie (37). Questi farmaci presentano anche importanti effetti a lungo termine in termini di riduzione del rischio cardiovascolare, come evidenziato dai primi risultati sullo studio EMPA-REG Outcome, effetti che per la prima volta vengono attribuiti a un farmaco ipoglicemizzante orale (38). Lo studio CANVAS (Canaglifozin Cardiovascular Assessment Study) ha confrontato Canagliflozin, altro inibitore SGLT2, con placebo e terapia standard in una coorte di 10.000 pazienti affetti da diabete mellito tipo 2 e con fattori di rischio e precedenti eventi cardiovascolari (CV). L'aggiunta di Canaglifozin è stata associata a una riduzione di morte $\mathrm{CV}$, infarto e ictus non fatale e di ospedalizzazione per scompenso cardiaco. Purtroppo, durante il trattamento con Canaglifozin, è stato riscontrato un aumentato rischio di amputazione delle dita degli arti inferiori (39).

Gli effetti cardio- e nefro-protettivi delle glifozine sono stati osservati nel trial multicentrico CREDENCE, in cui sono stati arruolati pazienti con diabete mellito tipo 2 e nefropatia diabetica. I meccanismi protettivi sono sia di tipo metabolico (dovuti alla lipolisi) che cardio-emodinamici (dovuti al miglioramento sia sul precarico che sul postcarico) (40). Attualmente, l'uso delle glifozine non è approvato in pazienti con GFR $<45 \mathrm{~mL} / \mathrm{min} / 1,73 \mathrm{~m}^{2}$, in considerazione del fatto che, con bassi valori di filtrato glomerulare, si ha una perdita della loro efficacia ipoglicemizzante (41).

\section{- La medicina di precisione e il periodo di transizione che porta all'inizio della terapia emodialitica}

Il concetto di medicina di precisione, oltre che nell'ambito delle singole patologie renali, negli ultimi anni ha assunto una particolare rilevanza, anche nella scelta delle cure nel periodo di transizione dalla malattia renale cronica in fase terminale al trattamento emodialitico. L'approccio personalizzato, che, complessivamente, considera la storia clinica del paziente, i fattori legati allo stile vita (dieta, attività fisica, eventuali situazioni di stress) e le preferenze personali, è essenziale nello stabilire quale strategia di trattamento (come emodialisi versus dialisi peritoneale, trapianto di rene, terapia conservativa) è più adatta per il singolo individuo (42) (Fig. 5). Date le molte incertezze che riguardano l'ottimale percorso di transizione da seguire nel passaggio dalla malattia renale cronica nella sua fase terminale alla dialisi piuttosto che al trapianto, sarebbe opportuno migliorare l'applicazione della medicina di precisione anche in questa popolazione. Future ricerche, infatti, sono necessarie per identificare terapie aggiuntive che promuovano un regime dialitico incrementale. La dialisi incrementale necessita di stabilire un ben definito programma terapeutico e richiede un'importante attenzione clinica. La dialisi incrementale inizia dall'ambulatorio pre-dialisi nello spazio di tempo in cui il paziente con eGFR di 5-10 mL/ $\mathrm{min} / 1,73 \mathrm{~m}^{2}$ passa dalla terapia conservativa a una dieta 

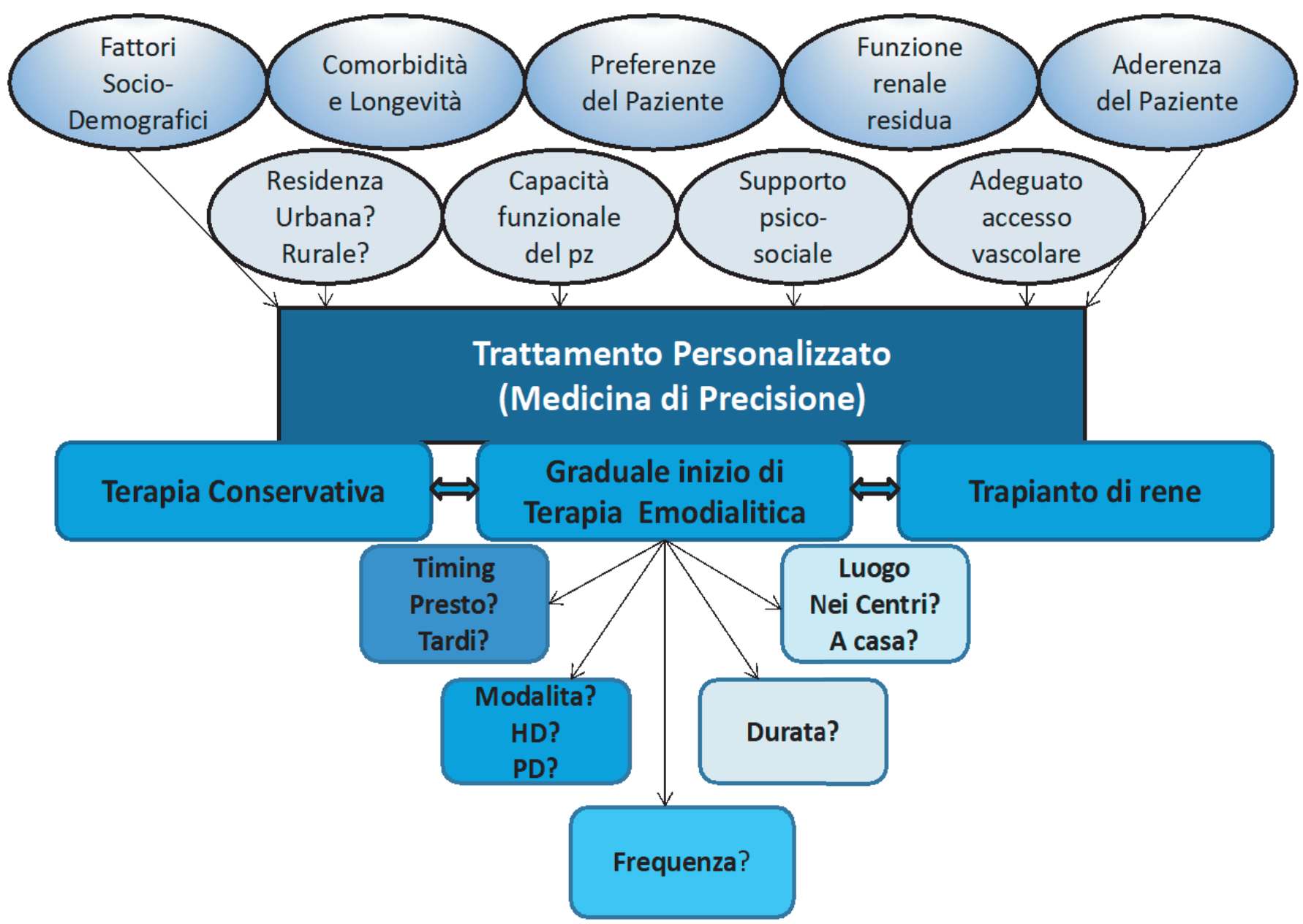

Fig. 5 - Approccio personalizzato nel periodo di transizione che porta all'inizio della terapia emodialitica.

ipoproteica integrata da una dialisi mono-settimanale e questo programma adattato al trend della funzione renale residua (FRR) (43). II rene in fase terminale conserva delle caratteristiche interessanti, tra cui la capacità di eliminare molecole altamente tossiche e a elevato peso molecolare, come le protein bound uremic toxins (PBUT), come l'indossil solfato e come il p-cresolo solfato, che si legano stabilmente all'albumina (44). L'emodialisi e i trattamenti ad alta ultrafiltrazione riescono a eliminare solo la parte protein-free delle PBUT, che rappresenta il $10 \%$ del loro quantitativo totale (45). Cercando di mantenere una buona diuresi residua, si riescono a ottenere delle perdite urinarie di fosfati e questo grazie all'abbassamento della soglia tubulare dei fosfati. Oltre a privilegiare la FRR, occorre anche aggiungere la prescrizione di una dieta ipoproteica, perché riesce a risparmiare nefroni, rallentando l'inizio della terapia sostitutiva.

Da qui, si rinforzano le basi per iniziare un trattamento emodialitico incrementale (46).

I pazienti sottoposti a trapianto renale vanno incontro a un miglioramento della loro qualità di vita e della sopravvivenza rispetto alla dialisi. II complesso processo, che va dalla valutazione del candidato all'intervento chirurgico di trapianto, all'inizio della terapia immunosoppressiva e alla sua scelta e alle cure di follow-up dopo il trapianto è rigorosamente strutturato e regolamentato. L'approccio personalizzato al trapianto di rene, come prevede l'uso della medicina di precisione, richiede una combinazione di linee guida consolidate, nozioni emergenti, nuove terapie e perfezionamento delle cure (47).

\section{Conclusioni e aspetti futuri}

Questa review analizza lo stato delle scoperte scientifiche sulla genomica e sulle sue attuali applicazioni diagnostiche e terapeutiche ed evidenzia quali sono le aree dove le scoperte genetiche sono già utilizzate nella pratica clinica. È utile, per tale motivo, che si sviluppino nuove conoscenze in materia e che la genetica clinica acquisisca un ruolo maggiore nella pratica clinica di tutti i medici. La medicina di precisione rappresenta un importante step nell'evoluzione 
delle cure mediche e ha illuminato il nostro modo di curare i pazienti permettendo di studiare i meccanismi patogenetici alla base di una malattia e, potenzialmente, di trovarne una cura. C'è la speranza che la medicina di precisione possa individuare nuovi farmaci, che agiscano in maniera mirata sulle alterazioni genetiche presenti nell'ambito di patologie nefrologiche e non, che non hanno ancora avuto una cura.

\section{Disclosures}

Conflict of interest: The authors declare no conflict of interest. Financial support: This research received no specific grant from any funding agency in the public, commercial, or not-for-profit sectors.

\section{Bibliografia}

1. Annovi G. Cos'è la medicina di precisione. Forward. 2016. Online

2. Torrisi AM, Granata A. Indicatori bibliometrici delle riviste di Nefrologia: criticità e pregi. G Ital Nefrol 2016;33:1-18. Online

3. Gameiro GR, Sinkunas V, Liguori GR, Auler-Júnior JOC. Precision Medicine: changing the way we think about healthcare. Clinics (São Paulo). 2018;73:e723. CrossRef PubMed

4. Bittencourt MS. From Evidence-Based Medicine to Precision Health: Using Data to Personalize Care. Arq Bras Cardiol. 2018;111(6):762-763. CrossRef PubMed

5. Issa AM. Personalized medicine and the practice of medicine in the 21st century. McGill J Med. 2007;10(1):53-57. PubMed

6. Vogel F. Moderne problem der humangenetik. Ergeb Inn Med U Kinderheilk. 1959;12:52-125.

7. Pulciani S, Di Lonardo A, Fagnani C, Taruscio D. P4 Medicine versus Hippocrates. Ann Ist Super Sanita. 2017;53(3):185191. PubMed

8. Pecci A, Panza E, Pujol-Moix N, et al. Position of nonmuscle myosin heavy chain IIA (NMMHC-IIA) mutations predicts the natural history of MYH9-related disease. Hum Mutat. 2008;29(3):409-417. CrossRef PubMed

9. Psaty BM, Dekkers OM, Cooper RS. Comparison of 2 Treatment Models: Precision Medicine and Preventive Medicine. JAMA. 2018;320(8):751-752. CrossRef PubMed

10. Granata M, Canto C, Mazzarino MC, Fatuzzo P, Granata A. [Which genetic testing in renal disease]. G Ital Nefrol. 2015; 32(5):gin/32.5.3. PubMed

11. Collins FS. Shattuck lecture-medical and societal consequences of the Human Genome Project. N Engl J Med. 1999; 341(1):28-37. CrossRef PubMed

12. National Research Council (US) committee on a Framework for Development a New Taxonomy of Disease. Toward Precision Medicine: Building a knowledge network for biomedical research and a new taxonomy of disease Washington (DC): National Academies Press (US); 2011. pp 142. Online (Accessed July 2021)

13. Barbagallo C, Passanisi R, Mirabella F, et al. Upregulated microRNAs in membranous glomerulonephropathy are associated with significant downregulation of IL6 and MYC mRNAs. J Cell Physiol. 2019;234(8):12625-12636. CrossRef PubMed

14. Goutsouliak K, Veeraraghavan J, Sethunath V, et al. Towards personalized treatment for early stage HER2-positive breast cancer. Nat Rev Clin Oncol. 2020;17(4):233-250. CrossRef PubMed

15. Delgado-Goni T, Miniotis MF, Wantuch S, et al. The BRAF inibitor vemurafenib activates mitochondrial metabolism and inhibits hyperpolarized pyruvate-lactate exchang in BRAF mutat human melanoma cells. Mol Cancer Ther. 2016;15(12):29872999. CrossRef PubMed

16. Mendell J, Freeman DJ, Feng W, et al. Clinical Translation and validation of a Predictive biomarker for Patritumab, an antihuman Epidermal Growth Factor Receptor 3 (HER3) monoclonal antibody, in patients with advanced non-small cell lung cancer. EBioMedicine. 2015;2(3):264-271. CrossRef PubMed

17. Dugger SA, Platt A, Goldstein DB. Drug development in the era of precision medicine. Nat Rev Drug Discov. 2018;17(3):183196. CrossRef PubMed

18. Kim JE, Kim H-E, Park Jl, et al. The association between gut microbiota and uremia of Chronic Kidney Disease. Microorganisms. 2020;8(6):907. CrossRef PubMed

19. Vaziri ND, Wong J, Pahl M, et al. Chronic kidney disease alters intestinal microbial flora. Kidney Int. 2013;83(2):308315. CrossRef PubMed

20. Kim HE, Kim JE, Park Jl, et al. SAT 184 The potential function of gut bacteria, Oscillibacter, on the uremia of chronic kidney disease patients. Kidney Int Rep. 2020;5(3):S78. CrossRef

21. Gong J, Noel S, Pluznick JL, Hamad ARA, Rabb H. Gut Microbiota-Kidney Cross-Talk in Acute Kidney Injury. Semin Nephrol. 2019;39(1):107-116. CrossRef PubMed

22. Kuo IY, Chapman AB. Polycystins, ADPKD, and cardiovascular disease. Kidney Int Rep 2019;5:396-406. CrossRef PubMed

23. Yamaguchi T, Hempson SJ, Reif GA, Hedge AM, Wallace DP. Calcium restores a normal proliferation phenotype in human polycystic kidney disease epithelial cells. J Am Soc Nephrol. 2006;17(1):178-187. CrossRef PubMed

24. Yang B, Sonawane ND, Zhao D, Somlo S, Verkman AS. Smallmolecule CFTR inhibitors slow cyst growth in polycystic kidney disease. J Am Soc Nephrol. 2008;19(7):1300-1310. CrossRef PubMed

25. Torres VE, Higashihara E, Devuyst O, et al; TEMPO 3:4 Trial Investigators. Effect of Tolvaptan in Autosomal Dominant Polycystic Kidney Disease by CKD Stage: Results from the TEMPO 3:4 Trial. Clin J Am Soc Nephrol. 2016;11(5):803-811. CrossRef PubMed

26. Perico N, Remuzzi G. Trattamento della malattia policistica autosomica dominante del rene (ADPKD): analoghi della somatostatina e inibitori mTOR. G Ital Nefrol. 2016;33:1-12. Online

27. Pyronnet S, Bousquet C, Najib S, Azar R, Laklai H, Susini C. Antitumor effects of somatostatin. Mol Cell Endocrinol. 2008;286(1-2):230-237. CrossRef PubMed

28. Perico N, Ruggenenti P, Perna A, et al; ALADIN 2 Study Group. Octreotide-LAR in later-stage autosomal dominant polycystic kidney disease (ALADIN 2): A randomized, double-blind, placebo-controlled, multicenter trial. PLoS Med. 2019;16(4): e1002777. CrossRef PubMed

29. Torres VE, Harris PC. Mechanisms of Disease: autosomal dominant and recessive polycystic kidney disease. Nat Clin Pract Nephrol. 2006;2(1):40-55. PubMed

30. Ibraghimov-Beskrovnaya $O$, Natoli TA. mTOR signaling in polycystic kidney disease. Trends Mol Med. 2011;17(11):625633. CrossRef PubMed

31. Shillingford JM, Murcia NS, Larson $\mathrm{CH}$, et al. The mTOR pathway is regulated by polycystin-1, and its inhibition reverses renal cystogenesis in polycystic kidney disease. Proc Natl Acad Sci USA. 2006;103(14):5466-5471. CrossRef PubMed

32. Moes DJ, Guchelaar HJ. De Fijter JW. Sirolimus and everolimus in kidney transplantation. Drug Discov Today 2015;20:1243 1249. CrossRef PubMed

33. Santoro D, Pellicanò, Visconti L, et al. Nuove opzioni terapeutiche per la malattia renale policistica. Giornale di Tecniche Nefrologiche e Dialitiche. 2016;28(2):143-152. CrossRef 
34. Gnavi R, Migliardi A, Maggini M, Costa G. Prevalence of and secular trends in diagnosed diabetes in Italy: 1980-2013. Nutr Metab Cardiovasc Dis. 2018;28(3):219-225. CrossRef PubMed

35. Pintaudi B. Gli standard Italiani 2018 per la terapia del diabete mellito. Giornale Italiano di Farmacoeconomia e Farmacoutilizzazione 2018;10:5-14. Online

36. Iacoviello M, Marini M, Benvenuto M et al. Programma dell'Area Scompenso Cardiaco per il biennio 2019-2020. Cardiologia negli Ospedali. 2019;(228):51-52. Online

37. Zanoli L, Granata A, Lentini $P$, et al. Sodium-glucose linked transporter-2 inhibitors in chronic kidney disease. Scientific World Journal. 2015;2015:317507. CrossRef PubMed

38. Verma S, McMurray JJV. The Serendipitous Story of SGLT2 Inhibitors in Heart Failure. Circulation. 2019;139(22):25372541. CrossRef PubMed

39. Greco EV, Bacci S. Impatto dei nuovi farmaci antidiabetici nella storia naturale della nefropatia. Giornale Italiano di Diabetologia e Metabolismo. 2018;38:104-114.

40. Zelniker TA, Wiviott SD, Raz I, et al. SGLT2 inhibitors for primary and secondary prevention of cardiovascular and renal outcomes in type 2 diabetes: a systematic review and meta-analysis of cardiovascular outcome trials. Lancet. 2019;393(10166):3139. CrossRef PubMed
41. Insalaco M, Zanoli L, Rastelli S, et al. [Sodium Glucose Cotransporter Type 2 (SGLT2) Inhibitors in CKD]. G Ital Nefrol. 2015;32(4):gin/32.4.2. PubMed

42. Rhee CM, Obi Y, Mathew AT, Kalantar-Zadeh K. Precision Medicine in the transition to Dialysis and personalized Renal Replacement Therapy. Semin Nephrol. 2018;38:325-335. CrossRef PubMed

43. Bolasco P, Murtas S. Emodialisi infrequente e incrementale: differenze e definizioni. G Ital Nefrol. 2018;35:1-8. Online

44. Vanholder R, Schepers E, Pletinck A, Nagler EV, Glorieux G. The uremic toxicity of indoxyl sulfate and p-cresyl sulfate: a systematic review. J Am Soc Nephrol. 2014;25(9):1897-1907. CrossRef PubMed

45. Glorieux G, Vanholder R. New uremic toxins - which solutes should be removed? Contrib Nephrol. 2011;168:117-128. CrossRef PubMed

46. Locatelli F, Del Vecchio L, Aicardi V. Nutritional Issues with incremental dialysis: The role of low Protein Diets. Semin Dial. 2017;30(3):246-250. CrossRef PubMed

47. Donald C. Dafoe, Ekamol Tantisattamo. Precisione Medicine and Personalized Approach to Renal Transplantation. Semin Nephrology Vol 38 Luglio 2018 pg 346-354. CrossRef PubMed 